\title{
Berliner ophthalmologische Gesellschaft
}

\section{Sitzung vom 16. Juni 1904}

Vorsitzender: Herr v. Michel. Schriftführer: Herr Wertheim.

Пerr Herzog berichtet über seine Studien an der Netzhaut, die ilm zu der Anschauung führten, dass dieselbe sich wie eine poröse Membran verhält, durch welche Kräfte im Sinne eines osmotischen Druckes einwirken. Wenn man ein frisches L·1/80schauge in HN03-Lösung auf eine viertel Stunde bringt, dann das Auge üffnet, den Giaskörper in destilliertem Wasser leicht entfernt, so sieht man nach 10-15 Minuten transparente Bläschen aufschiessen. In HN03 wiederum verschwinden die Bläschen spurlos, ein Spiel, das beliebig zu wiederholen ist. Die niikroskopische Untersuchung so behandelter Netzhäute ergab nun eine Spaltung der inneren Körnerschichten, keine wirkliche Abhebung. Pigment und Zapfen befinden sich in Lichtstellung; bei Nachtfröschen hingegen unterblieb die Spaltung, und es trat reguläre Abhebung ein. Daraus ergibt sich also nach Herzog folgendes: 1. Bei Isotonie wirken starke Kräfte bis zur Spaltung der inneren Schichten; 2. der Belichtungszustand sichert die Anhaftung der Netzhaut; 3. die Netzhaut verhält sich wie eine poröse Stempelwand. Ob diese poröse Eigenschaft auch der normalen und lebenden Netzhaut zukommt, lässt H. unentschíeden, zumal am Lebenden solche Experimente nicht wohl zu machen sind.

In der Diskussion meint Herr $\Lambda$ Vessely, dass die lebende Netzhaut eine permeable Membran ist; es handele sich bei den Versuchen II.'s nicht um einen osmotischen Prozess, sondern urn einen Wirbelstrom.

Offene Korrespondenz.

87

Hen- Ginsberg maclit darauf aufmerksam, dass sich bei Ablösung der Netzliaut bei holier Myopie oft eine ganz entsprechende Trennung in den Retinasctiichten findet.

Herr Abels dor ff meint, dass sich die Froschexperimente H.'s weder avf den Menschen, noch überhaupt auf das Säugetierauge iiber-trage $\pi$ lassen.

Im Schlusswort zweifelt Herr Herzog nicht an der osmotischen Natur seiner Versuche. Herr v. Michel demonstriert alsdann zwei Fälle von Myasthenia gravis pseudoparaly tica, und zwar von rein okularer Form mit vor-zugsweisem Ergriffenseiø des Muse, levator palpebr. super. Die Patienten sind 5 resp. 47 Jahre alt. Bei dem Knaben findet sich die myasthenische Reaktion auch im Orbicularis, der M. ciliaris und Sphinkter sind nicht gestört. Die Ptosis wird bei dem Knaben besonders stark, wenn der-selbe durch Laufen müde wird. Pathologisch-anatomische Beíunde solcher Fälle liegen noch sehr spärlich vor; Oppenheim fand eiue Ausdehnung des IV. Ventrikels ausgesprochen. Vielleícht handelt es sich auch um eine Toxinwirkung. In der Diskussion bemerkt Herr Loeser, dass er in Oppenheims Poliklinik mehrere Fälle von Myasthenia gravis pseudoparalytica mit Morb. Basedowii kombiniert beobachtet habe, was wohl kaum ein Zufall ware; der Morb. Basedowii s chaff e vielleicht die Disposition für die Myasthenie. Therapeutisch sei nach richtig gestellter Diagnose vor Hg. etc. zu warnen. Herr v. Michel demonstriert zwei Fälle von Tuberkalose des Auges, und zwar erstens einen lljährigen Knaben mit abgelaafener Keratitis parenchymatosa und Iritis, wobei die Tuberkulin- 
Injektion positiv austiel, zweitens einen erwachsenen Mann, bei welchem ausser einseitiger frischer Neuritis retrobulbaris in der Macula lutea drei kleine helle Chorioidealpunkte sich fanden (zentrales Skotom!), in den äqua-torialen Partieu der Retina kleine gelbliche punktartige Trübungen. Herr v. Michel diagnostizierte eine tuberkulöse Neuritis optica. Auch hier fiel die Tuberkulinprobe positiv (Temperatur 39, $8^{\circ}$ ) aus, es handelte sich also um lokale Augentuberkulose. Sonst fand sich nur noch germ ger Spitzenkatarrh.

Zum Schlusse demonstriert Herr v. Michel mittels des Epidiaskops die Ausbreitung der elastischen Fasern im Naevus, sowie mikroskopische Präparate von Tuberkulöse des Auges. Pollack.

Offene Korrespondenz.

Budapest.G. Gesellschaft ungariseher Augenärzte. In Budapest beschlossen die Augenärzte Ungarns am 22. Mai 1. Js. die Gründung einer ophthaimologisehen Gesellschaft nach Muster der „Heidelberger ophthalmo-logischen Gesellschaft" und der „Société française d'ophthalmologie”. An der Besprechung nahmen 30 Augenärzte teil

ihre Zustimmung gaben weitere 16 Kollegen. In das Komitee zur Vorbereitung des nächsten Kongresses 